APPEARED IN BULLETIN OF THE

AMERICAN MATHEMATICAL SOCIETY

Volume 28, Number 1, January 1993, Pages 109-115

\title{
SINGULARITIES OF THE RADON TRANSFORM
}

\author{
A. G. Ramm and A. I. Zaslavsky
}

\begin{abstract}
Singularities of the Radon transform of a piecewise smooth function $f(x), x \in R^{n}, n \geq 2$, are calculated. If the singularities of the Radon transform are known, then the equations of the surfaces of discontinuity of $f(x)$ are calculated by applying the Legendre transform to the functions, which appear in the equations of the discontinuity surfaces of the Radon transform of $f(x)$; examples are given. Numerical aspects of the problem of finding discontinuities of $f(x)$, given the discontinuities of its Radon transform, are discussed.
\end{abstract}

\section{INTRODUCTION}

Let $f(x)$ be a compactly supported function, $D$ be its support, and $\Gamma=\partial D$ be a union of finitely many $C^{\infty}$ hypersurfaces $\Gamma_{1}, \ldots, \Gamma_{s}$ in general position, each of which can be written in local coordinates as

$$
x_{n}=g\left(x^{\prime}\right), \quad x^{\prime}=\left(x_{1}, \ldots, x_{n-1}\right), n \geq 2,
$$

where $g\left(x^{\prime}\right) \in C^{\infty}, f(x) \in C^{\infty}(D),\left.f(x)\right|_{\Gamma} \geq c>0$. The discontinuity surface of $f(x)$ is $\Gamma$, the boundary of $D$. We assume that the rank of the Hessian $g_{i j}(x):=$ $\partial^{2} g / \partial x_{i} \partial x_{j}$ is constant on each of $\Gamma_{j}, 1 \leq j \leq s$.

Define the Radon transform (RT) of $f(x)$ by the usual formula [GGV] $\hat{f}(p, \alpha)=$ $\int_{\mathbb{R}^{n}} f(x) \delta(p-\alpha \cdot x) d x$, where $\delta$ is the delta-function. It is well known that $\hat{f}(\lambda p, \lambda \alpha)=$ $|\lambda|^{-1} \hat{f}(p, \alpha), \lambda \in R^{1}, \lambda \neq 0$. Consider the integral

$$
R(p, \alpha ; f):=\int_{l_{\alpha p}} f(x) \mu(d x),
$$

where $l_{\alpha p}$ is the plane $\alpha \cdot x-p=0, \alpha \in \mathbb{R}^{n}, p \in \mathbb{R}^{1}$, and $\mu(d x)$ is the Lebesgue measure on $l_{\alpha p}$. One has $R(p, \alpha ; f)=\hat{f}\left(p /|\alpha|, \alpha^{0}\right), \alpha^{0}:=\alpha|\alpha|^{-1}$, so that $R(p, \alpha ; f)=$ $|\alpha| \hat{f}(p, \alpha),|\alpha|=\left(\alpha_{1}^{2}+\cdots+\alpha_{n}^{2}\right)^{1 / 2}$.

The problems we are interested in are: (P1) Find the singularities of $R(p, \alpha ; f)$; and (P2) Find the surface $\Gamma$ of discontinuity of $f(x)$ given the singularities of $R(p, \alpha ; f)$.

No results concerning (P2) were known. In $[\mathrm{N}]$ one can find an estimate of the norm of $\hat{f}(p, \alpha)$ in Sobolev spaces. This result does not give information about (P1) and $(\mathrm{P} 2)$. In $[\mathrm{P}]$ there is a result given without proof, which has a relation to $(\mathrm{P} 1)$. Our result is more general. In $[Q]$ it is mentioned that the values $(\alpha: p)$, such that

1991 Mathematics Subject Classification. Primary 44A12.

Received by the editors March 11, 1992 
$l_{\alpha p}$ is tangent to $\Gamma$, play a special role. This observation is made quantitative in our Theorem 1. Our results are useful for inversion of incomplete tomographic data $[R 2]$.

The basic results are formulated in $\S$ II. They give solutions of the problems (P1) and (P2). Actually, more general problems are solved; particularly, finite smoothness of $f(x)$ and $\Gamma$ is allowed, the role of the intersections of $\Gamma_{j}$ in the study of the singularities of $R(p, \alpha ; f)$ is clarified, etc. In $\S I I I$ proofs are sketched. In $\S \mathrm{IV}$ examples are given. In $\S \mathrm{V}$ numerical aspects of problem (P2) are discussed.

We conclude this introduction by an outline of our ideas. First, we describe the behavior of $R(p, \alpha ; f)$ in a neighborhood of the set $Q_{f}$ which is the set of singularities of $R(p, \alpha ; f)$. Second, we prove that, in general, there is an equation of the set $Q_{f}$ which is of the form $q=h(\beta), \beta \in R^{n-1}$, so that $Q_{f}$ is a hypersurface. Third, we prove that the function $g\left(x^{\prime}\right)$ (in the equation of $\Gamma$ ) is the Legendre transform of the function $h(\beta)$ (in the equation of $Q_{f}$ ). Fourth, we describe some geometric properties of $Q_{f}$.

Our results give a theoretical basis for the solution of the practically important problem in nondestructive evaluation and remote sensing, the problem of finding the discontinuities of a function from the knowledge of its RT.

\section{Formulation of the Results}

The RT, defined by formula (1), is a function on the projective space $\mathbb{R} \mathbb{P}_{n}$, and we take $R(1,0 ; f):=0$ for compactly supported $f$. Let $Q_{f}$ denote the set of the points $(\alpha: p)$ in this projective space, which correspond to the planes $l_{\alpha p}$ tangent to $\Gamma=\partial D$. We say that $l_{\alpha p}$ is tangent to $\Gamma$ at a point $x \in B_{m}:=\bigcap_{j=1}^{m} \Gamma_{j}$, if $l_{\alpha p}$ is not transversal to $B_{m}$ at the point $x$.

1. Our first result is the following theorem in which the description of the singularities of $R(p, \alpha ; f)$ is given.

Let $l_{\alpha p}$ be tangent to $\Gamma$ at the point $\bar{x}$. We claim that if $\bar{\alpha}$ is generic, then the set $Q_{f}$ is a smooth hypersurface in a neighborhood $U$ of $(\bar{\alpha}: \bar{p})$. If $A$ is a symmetric matrix with real-valued entries, then its inertia index (inerdex) is defined to be the number of its negative eigenvalues. Consider first the case when $\Gamma$ consists of one surface.

Theorem 1. There exists an equation $\zeta(\alpha: p)=0, \nabla \zeta \neq 0$ in $U$, which defines $Q_{f}$ in $U$, and two $C^{\infty}$ functions $r_{1}$ and $r_{2}$ in $U$ such that

$$
R(p, \alpha ; f)= \begin{cases}\zeta_{+}^{(n-1) / 2} r_{1}+r_{2}, & \text { if } \text { In is even }, \\ \zeta^{(n-1) / 2}(\ln |\zeta|) r_{1}+r_{2}, & \text { if } \text { In is odd. }\end{cases}
$$

Here $I$ is the inerdex of the matrix $z_{k j}$, where $z_{k j}$ is the Hessian of the function $z=(\bar{\alpha} \cdot x-\bar{p}) /|\bar{\alpha}|$ on $\Gamma$ at the point $\bar{x}$ and $z_{+}=\max (z, 0)$.

If $\bar{x} \in B_{m}$ and $(\bar{\alpha}: \bar{p})$ is generic, then the following result holds.

Theorem $1^{\prime}$. There exists $\zeta(\alpha: p), \nabla \zeta \neq 0$ in $U$, such that the equation $\zeta(\alpha: p)=$ 0 is the equation of $Q_{f}$ in $U$, and two $C^{\infty}$ functions $r_{1}$ and $r_{2}$ in $U$, such that

$$
R(p, \alpha ; f)= \begin{cases}\zeta_{+}^{(n+m-2) / 2} r_{1}+r_{2}, & \text { if } I(n+m-1) \text { is even }, \\ \zeta^{(n+m-2) / 2}(\ln |\zeta|) r_{1}+r_{2}, & \text { if } I(n+m-1) \text { is odd. }\end{cases}
$$


In [RZ1] the constant $r_{1}(\bar{\alpha}: \bar{p})$ is calculated. In [RZ2] this result is used for a derivation of the asymptotics of the Fourier transform of a piecewise smooth function.

2. Let us define the Legendre transform of a function $g(y), y \in R^{n-1}$ in a neighborhood $U_{\bar{y}}$ of a point $\bar{y}$ at which the matrix $g_{i j}(y):=\partial^{2} g / \partial y_{i} \partial y_{j}$ is nondegenerate, i.e., $\operatorname{det} g_{i j}(y) \neq 0$ in $U_{\bar{y}}$. Define $L g:=h(\beta):=\beta \cdot y-g(y)$, where the dot stands for the inner product and $y=y(\beta)$ is the unique solution of the equation $\beta=\nabla g(y)$ in a neighborhood $U_{\bar{\beta}}$ of the point $\bar{\beta}=\nabla g(\bar{y})$. One can prove that if $g \in C^{l}\left(U_{\bar{y}}\right)$, $l \geq 2$, and $\operatorname{det} g_{i j}(y) \neq 0$ in $U_{\bar{y}}$, then $h(\beta) \in C^{l}\left(U_{\bar{\beta}}\right)$.

It is known that under our assumptions $L h=g(y)$, i.e., the Legendre transform is involutive: $g(y)=\beta \cdot y-h(\beta)$, where $\beta=\beta(y)$ is the unique solution to the equation $y=\nabla h(\beta), \beta \in U_{\bar{\beta}}$. One can prove that $\operatorname{det} h_{i j}(\beta) \neq 0$ in $U_{\bar{\beta}}$ if $\operatorname{det} g_{i j}(y) \neq 0$ in $U_{\bar{y}} ;$ moreover, the matrix $h_{i j}(\beta)$ is inverse to $g_{i j}(y)$, where $\beta=\beta(y)$. Recall that $\Gamma$ is a union of hypersurfaces $\Gamma_{j}, 1 \leq j \leq s, \Gamma_{1}, \ldots, \Gamma_{s}$ are $C^{\infty}$ and in general position. Denote $\widehat{B}_{m}:=\Gamma_{1, \ldots, m}$ the set of $(\alpha: p) \in \mathbb{R} \mathbb{P}_{n}$ such that $l_{\alpha p}$ is tangent to $B_{m}$. The set $\widehat{B}_{m} \subset \mathbb{R P}_{n}$ may not be a hypersurface (see Theorem 3 ); however, as Theorem $1^{\prime}$ claims, it is indeed a smooth hypersurface outside a set of $(n-1)$-dimensional Lebesgue's measure zero.

3. Our second result gives the relation between the discontinuity surfaces for $R(p, \alpha ; f)$ and those for $f(x)$; namely, the function $g\left(x^{\prime}\right)$ in the local equation of $\Gamma, x_{n}=g\left(x^{\prime}\right)$, is the Legendre transform of the function $h(\beta)$ which gives the equation of $Q_{f}$, $q=h(\beta)$.

Assume that $q=h(\beta), \beta \in U_{\bar{\beta}}$, where $U_{\bar{\beta}}$ is a neighborhood of a point $\bar{\beta}$, $\bar{q}=h(\bar{\beta})$, and $\operatorname{det} h_{i j}(\beta) \neq 0$ in $U_{\bar{\beta}}$, where $h_{i j}:=\partial^{2} h / \partial \beta_{i} \partial \beta_{j}$. Let $\bar{x}^{\prime}=\nabla h(\bar{\beta})$.

Theorem 2. If $h(\beta) \in C^{l}\left(U_{\bar{\beta}}\right), l \geq 2$, then $L h=g\left(x^{\prime}\right)$, and $g\left(x^{\prime}\right) \in C^{l}\left(U_{\bar{x}^{\prime}}\right)$.

This result allows one to recover the surfaces of discontinuity of $f(x)$ given the surfaces of discontinuity of $R(p, \alpha ; f)$.

4. Examples show that the Legendre transform $h(\beta)=L g$ of a function $g\left(x^{\prime}\right)$, $x^{\prime} \in \mathbb{R}^{n-1}$, may have domain of definition of dimension less than $n-1$. Since $Q_{f}$ is a union of several varieties of codimension one in $\mathbb{R P}_{n}$ (called components below), the question arises: which of the components of $Q_{f}$ and which of their intersections provide, after applying the generalized Legendre transform defined in [RZ1], parts of $\Gamma=\partial D$ which have codimension one in $\mathbb{R}^{n}$. The answer is given in Theorem 3. This theorem describes $Q_{f}$ in terms of differential geometry of $\Gamma$. Recall that the principal curvatures of a hypersurface $S \subset \mathbb{R}^{n}$, which is the graph of a function $x_{n}=g\left(x^{\prime}\right)$, are the eigenvalues of the matrix $\left(g_{i j}\right) \cdot\left(\delta_{i j}+g_{i} g_{j}\right)^{-1} \cdot\left(1+\sum_{i=1}^{n-1} g_{i}^{2}\right)^{-1 / 2}$, $g_{i}=\partial g / \partial x_{i}$. One can prove that if $k, k \geq 1$, principal curvatures of a hypersurface $S$ vanish identically, then for every point $P \in S$ there exists an affine $k$-dimensional space $L_{P}$ such that $P \in L_{P} \subset S$.

Theorem 3. (a) Assume that $B_{m}$ is nonempty. Then $m$ principal curvatures of $\widehat{B}_{m}$ vanish identically;

(b) If $k$ principal curvatures of $\Gamma_{1}$ vanish identically, then $\widehat{\Gamma}_{1}$ has codimension $k+1$ in $\mathbb{R P}_{n}$.

Every point of $\widehat{\Gamma}_{1}$ is a vertex of a cone $K$, which belongs to $\Gamma_{1 j}$, where $\Gamma_{1} \cap \Gamma_{j} \neq$ $\varnothing$. The directrix of $K$ is $(k-1)$-dimensional, and this directrix can be described as 
follows: Take an arbitrary point $P \in \Gamma_{1}$, and let $L_{k}(P) \subseteq \Gamma_{1}$ be a $k$-dimensional affine space containing $P$, which exists since $k$ principal curvatures of $\Gamma_{1}$ vanish identically. Let $d_{P}:=\left\{(\alpha: p): l_{\alpha p}\right.$ be tangent to $\Gamma_{j}$ at the points of $\left.L_{K}(P) \cap \Gamma_{j}\right\}$, and let $l_{\alpha_{0} p_{0}}$ be tangent to $\Gamma_{1}$ at the point $P$. The vertex of $K$ is the point $\left(\alpha_{0}: p_{0}\right)$. The directrix of $K$ is the set $d_{P}$.

The set $Q_{f}$ is a union of the sets $\widehat{\Gamma}_{i_{1} \cdots i_{k}}, Q_{f}=\bigcup \widehat{\Gamma}_{i_{1} \cdots i_{k}}$ where the union is taken over all combinations of indices $1 \leq i_{k} \leq s$. Theorem 3 gives a recipe to select the components of $Q_{f}$ which yield after the Legendre transform the components of $\Gamma$ of codimension 1, i.e., hypersurfaces $\Gamma_{j}$ which are parts of $\Gamma, \Gamma=\bigcup_{j=1}^{s} \Gamma_{j}$. Note that if a component of $Q_{f}$ has some principal curvatures vanishing identically, then its preimage in $\mathbb{R}^{n}$ has codimension greater than one. Therefore, if one wishes to recover hypersurface-type components of $\Gamma$, then one should apply the Legendre transform to those components of $Q_{f}$, which do not have principal curvatures which vanish identically. Those hypersurfaces $\Gamma_{j}$ which have identically vanishing principal curvatures are reconstructed by applying the generalized Legendre transform, which was introduced in [RZ1], to high-codimension parts of $Q_{f}$ described in Theorem 3(b). The generalized Legendre transform was applied in $[\mathrm{Z}]$ to the study of dual varieties in algebraic geometry.

It is well known that the Radon transform may be considered as a Fourier integral operator, so it makes sense to study its action on the wave front set of $f$. In [RZ1] we study a relation of the wave front of $f$ and the set $Q_{f}$.

\section{Proofs of Theorems 1 And 2}

We sketch the proofs in the simplest case $m=1, n=2$, but the ideas are similar in the general case.

First we prove that if $D \subset C^{\infty}$ and $f \in C^{\infty}$, then $R(p, \alpha ; f) \in C^{\infty}$ on the set $V_{f}:=\mathbb{R P}_{n} \backslash Q_{f}$. Thus, the singularities of $f$ are in the set $Q_{f}$. Second, we prove that, generically, $Q_{f}$ is a $C^{\infty}$ hypersurface in $\mathbb{R P}_{n}$ and find the equation of this hypersurface.

Third, we prove that there exists a neighborhood $U$ of a generic point $(\bar{\alpha}: \bar{p})$ and an equation $\zeta(\alpha: p)=0, \nabla \zeta \neq 0$ in $U$, such that (2) holds.

(a) Let us start with the second claim and prove also Theorem 2 for $n \geq 2$. Let $\alpha \cdot x-p=0$ be a tangent plane $l_{\alpha p}$ to $\Gamma$ at a point $\bar{x} \in \Gamma$. Assume that $\alpha_{n} \neq 0$, and write $x_{n}=\beta \cdot x^{\prime}-q, \beta_{i}:=-\alpha_{i} / \alpha_{n}, q:=-p / \alpha_{n}, x^{\prime}=\left(x_{1}, \ldots, x_{n-1}\right)$. Let $x_{n}=g\left(x^{\prime}\right)$ be the equation of $\Gamma$ in a neighborhood $\bar{U}$ of $\bar{x}$, and $\operatorname{det} g_{i j}\left(\bar{x}^{\prime}\right) \neq 0$. Then $\nabla g\left(x^{\prime}\right)=\beta, q=\beta \cdot x^{\prime}-g\left(x^{\prime}\right)$. Thus $q=h(\beta):=L g$. The equation $q=h(\beta)$ is the equation of $Q_{f}$ in the inhomogeneous coordinates $(\beta, q)$. One can prove that if $q \in C^{s}(\bar{U}), s \geq 2$, and $\operatorname{det} g_{i j}\left(\bar{x}^{\prime}\right) \neq 0$, then $h \in C^{s}(U)$, where $U$ is a neighborhood of the point $(\bar{\beta}, \bar{q}), \nabla g\left(\bar{x}^{\prime}\right)=\bar{\beta}, \bar{q}=\bar{\beta} \cdot \bar{x}^{\prime}-g\left(\bar{x}^{\prime}\right)$. Since $L$ is involutive, $g=L h$. Theorem 2 is proved.

(b) Let us prove the first claim for $n=2$. Assume that $(\alpha: p) \in V_{f}$, i.e., $l_{\alpha p}$ is not tangential to $\Gamma$. Write $R(p, \alpha ; f)$ as

$$
J:=\int_{a_{1}(q, \beta)}^{a_{2}(q, \beta)} f\left(x_{1}, \beta x_{1}-q\right) d x_{1},
$$

where $a_{i}:=a_{i}(q, \beta)$ are the points of intersection of $l_{\alpha p}$ with $\Gamma$. The integral $J$ is a sum of the integrals over the intervals $\left(a_{1}, b\right),(b, c),\left(c, a_{2}\right)$, where $a_{1}<b<c<a_{2}$ 
and $b, c$ do not depend on $q, \beta$. Obviously the integral over $(b, c)$ is a $C^{l}$ function of $\beta$ and $q$ if $f \in C^{l}, l \geq 0$. The integrals over $\left(a_{1}, b\right)$ and $\left(c, a_{2}\right)$ are treated similarly.

Let us prove that the integral over $\left(a_{1}, b\right)$ is $C^{l}$ function of $q, \beta$ if $\Gamma, f \in C^{l}$, $l \geq 2$, and $l_{\alpha p}$ is transversal to $\Gamma$, that is, $\beta \neq g^{\prime}\left(a_{1}\right)$. It is sufficient to prove that $a_{1}(q, \beta) \in C^{l}$. The function $a_{1}(q, \beta)$ is the root of the equation $q=\beta a_{1}-g\left(a_{1}\right)$. By the transversality condition $\beta-g^{\prime}\left(a_{1}\right) \neq 0$. Thus, the implicit function theorem implies that the root $a_{1}(q, \beta) \in C^{l}$ if $g \in C^{l}$. The first claim is proved.

(c) Let us prove the last claim. Let $(\bar{\alpha}: \bar{p}) \in Q_{f}$ and $(\bar{\beta}, \bar{q})$ be the corresponding nonhomogeneous coordinates. For a generic $(\bar{\alpha}: \bar{p})$ the condition $g^{\prime \prime}\left(\bar{x}_{1}\right) \neq 0$ follows from the equation $g^{\prime}\left(\bar{x}_{1}\right)=\bar{\beta}$ and Sard's theorem. We can assume therefore that $g^{\prime \prime}\left(\bar{x}_{1}\right) \neq 0$. Consequently, the point $\bar{x}_{1}$ is a Morse-type (nondegenerate) critical point of the function $z:=\bar{\alpha} \cdot x-\bar{p}$ on $\Gamma \cap \bar{U}$, i.e., of the function $-\bar{\beta} x_{1}+g\left(x_{1}\right)+\bar{q}$. The part of integral (1) taken over the complement to $\bar{U}$ is a $C^{\infty}$-function of $(\alpha: p)$ according to $(b)$. It gives $r_{2}$ in formula (2). By the Morse lemma, there are coordinates $u_{1}, u_{2}$ such that the equation of $\Gamma$ in these coordinates is $u_{1}=0$, the region $D \cap \bar{U}$ is described by the inequality $u_{1} \geq 0$, and $z=u_{1}+u_{2}^{2}$ in $\bar{U}$. To study the singularity of $R(p, \alpha ; f)$, take a curve $\gamma$ which intersects $Q_{f}$ transversally, for instance, $\gamma=\{(\alpha: p): \alpha=\bar{\alpha}\}$. Parameter $p$ gives the position of a point on $\gamma$. On $l_{\bar{\alpha} p}$ one has $\bar{\alpha} \cdot x-p=0$ and $z=\bar{\alpha} \cdot x-\bar{p}$, so $z=p-\bar{p}$ on $l_{\bar{\alpha} p}$. Thus, $z$ can be used as a parameter which determines the position of a point on $\gamma$; therefore, the domain of integration in (1) can be described by the inequality $u_{1} \geq 0$ and the equation $z-u_{1}-u_{2}^{2}=0$. Thus, $z-u_{2}^{2}=u_{1}$, so $-z_{+}^{1 / 2} \leq u_{2} \leq z_{+}^{1 / 2}$ since $z=z_{+} \geq 0$ in the integration region. We have

$$
R(p, \bar{\alpha} ; f)=\int_{l_{\bar{\alpha} p}} f(x) \mu(d x)=\int_{l} f_{1}\left(u_{1}, u_{2}\right) \mu_{1}(d u)=\int_{-z_{+}^{1 / 2}}^{z_{+}^{1 / 2}} f_{2}\left(u_{2}, z_{+}\right) d u_{2}
$$

where $f_{2}\left(u_{2}, z\right)$ is a $C^{\infty}$-function, $l$ is the curve given by the equation $z-u_{1}-u_{2}^{2}=0$ and $u_{1} \geq 0, \mu_{1}(d u)$ comes from $\mu(d x)$ via the Morse lemma change of variables, and the last integral comes after an elimination of $u_{1}$. From this formula one derives (2). Indeed, write $f_{2}\left(u_{2}, z\right)$ as a sum of even $f_{\mathrm{e}}$ and odd $f_{\mathrm{o}}$ functions of $u_{2}$, $f_{e}\left(u_{2}, z\right) \in C^{\infty}, f_{o}\left(u_{2}, z\right) \in C^{\infty}$. Then the integral

$$
\int_{-z_{+}^{1 / 2}}^{z_{+}^{1 / 2}} f_{\mathrm{e}}\left(u_{2}, z_{+}\right) d u_{2}=z_{+}^{1 / 2} r_{1} \quad \text { and } \quad \int_{-z_{+}^{1 / 2}}^{z_{+}^{1 / 2}} f_{o}\left(u_{2}, z_{+}\right) d u_{2}=0
$$

where $r_{1} \in C^{\infty}$. The function $r_{2}$ in formula (2) vanishes if $\Gamma$ is strictly convex so that $l_{\bar{\alpha} p}$ intersects $\Gamma$ at two points only.

\section{Examples}

1. Let $f(x)=1,|x| \leq a, f(x)=0,|x|>a, x \in \mathbb{R}^{n}, n \geq 2, \hat{f}\left(p, \alpha_{0}\right)=2 \sqrt{a^{2}-p^{2}}$, $\alpha^{0}=\alpha|\alpha|^{-1}$. Thus $p^{2} /|\alpha|^{2}=a^{2}$ is the equation of $Q_{f}$. In $(\beta, q)$ coordinates the equation of $Q_{f}$ is $q= \pm a \sqrt{1+\beta^{2}}, \beta \in \mathbb{R}^{n-1}$. Thus $h(\beta)= \pm a \sqrt{1+\beta^{2}}$. By Theorem 2 the equation $x_{n}=g\left(x^{\prime}\right)$ of the surface of discontinuity of $f(x)$ is given by $g\left(x^{\prime}\right)=L h=\mp \sqrt{a^{2}-x^{\prime 2}}$. The equation $x_{n}= \pm \sqrt{a^{2}-x^{\prime 2}}$ defines the sphere $|x|=a$.

2. Let $f(x)=1, b \leq|x| \leq a, f(x)=0,|x|<b$ or $|x|>a, 0<b<a, n \geq 2$. Then $\hat{f}\left(p, \alpha^{0}\right)=2 \sqrt{a^{2}-p^{2}}, b \leq p \leq a ; \hat{f}\left(p, \alpha^{0}\right)=2\left(\sqrt{a^{2}-p^{2}}-\sqrt{b^{2}-p^{2}}\right), 0 \leq p \leq b ;$ 
$\hat{f}\left(p, \alpha^{0}\right)=0, p>a,\left|\alpha^{0}\right|=1$. Thus $p^{2}=|\alpha|^{2} a^{2}$ and $p^{2}=|\alpha|^{2} b^{2}$ are the equations of $Q_{f}$. Taking Legendre's transform yields the surfaces $|x|=a$ and $|x|=b$ of discontinuity of $f(x)$.

3. Consider $f(x)=0$ outside of the region $D$ bounded by $\Gamma$, where $\Gamma$ is the union of the curves $x_{2}=0$ and $x_{2}=x_{1}^{2}-1$, and let $f(x)=1, x \in D$. The $R(p, \alpha ; f)$ is a function whose support is bounded by the curves $q=\beta, q=-\beta$ from below, $q=\frac{1}{4} \beta^{2}+1$ in the interval $-2 \leq \beta \leq 2$, and $q=\beta, q=-\beta$ for $|\beta| \geq 2$ from above. One can check that on the lines $q= \pm \beta,-\infty<\beta<\infty$, the function $R(p, \alpha ; f)$ has a singularity of the type $|z|$ and on the parabola $q=\frac{1}{4} \beta^{2}+1$ it has the singularity of the type $z_{+}^{1 / 2}$. Applying Legendre's transform first to the function $q=\frac{1}{4} \beta^{2}+1$, $-2 \leq \beta \leq 2$, yields the parabola $x_{2}=x_{1}^{2}-1,-1 \leq x_{1} \leq 1$; and secondly, applying it to the functions $q= \pm \beta$ yields two points $x_{1}= \pm 1, x_{2}=0$. By Theorem 3 , the straignt line joining these two points also belongs to $\Gamma$. Thus $\Gamma$ is recovered.

\section{NUMERICAL ASPECTS}

The RT of $f(x)$ is usually given with an error. Hence, the first numerical problem is to calculate the function $h(\beta)$ which gives the equation of the set $Q_{f}$ of the singularities of RT given the noisy measurements of the RT. The second numerical problem is to calculate $L h=g\left(x^{\prime}\right)$. Calculation of the Legendre transform of a function $h(\beta)$ known with errors is a well-posed problem, at least in the case when $\operatorname{det} g_{i j}\left(x^{\prime}\right) \neq 0$. It is proved in [RSZ] that if a function $g_{\delta}\left(x^{\prime}\right)$ given such that $\left|g_{\delta}\left(x^{\prime}\right)-g\left(x^{\prime}\right)\right|<\delta, g_{\delta}\left(x^{\prime}\right)$ is not necessarily in $C^{2}$ but is continuous, then one can calculate $L g$ with the accuracy $O(\delta)$ as $\delta \rightarrow 0$. This means that a stable method is given in [RSZ] for calculating the Legendre transform of noisy data. See also [R5]. Our result in part 3 of $\S I I$ has an interesting connection with the envelopes theory $[\mathrm{T}, \mathrm{Zl}]$.

\section{ACKNOWLEDGMENTS}

A. G. Ramm thanks ONR, NSF, and USIEF for support. The research of A. I. Zaslavsky was supported in part by a grant from the Ministry of Science and the "Ma-agara"-special project for absorption of new immigrants, in the Department of Mathematics, Technion.

\section{REFERENCES}

[GGV] I. M. Gelfand, M. I. Graev and N. Ya. Vilenkin, Generalized functions, Integral Geometry and Problems of Representation Theory, vol. 5, Academic Press, New York, 1966.

[N] F. Natterer, The mathematics of computerized tomography, Teubner, Stuttgart, 1986.

[P] V. Palamodov, Some singular problems of tomography, Mathematical Problems of Tomography (I. Gelfand and S. Gindikin, eds.), Amer. Math. Soc., Providence, RI, 1990, pp. $123-150$.

[Q] E. Quinto, Tomographic reconstructions from incomplete data-numerical inversion of the exterior Radon transform, Inverse Problems 4 (1988), 867-876.

[R1] A. G. Ramm, Random fields estimation theory, Longman, New York, 1990.

[R2] , Inversion of limited-angle tomographic data, Comp. and Math. with Appl. 22 (1991), 101-112.

[R3] - On numerical differentiation, Izvestiya Vuzov Math. 11 (1968), 131-135.

[R4] Estimates of the derivatives of random functions, J. Math. Anal. Appl. 102 (1984), 244-250; (with T. Miller) 110 (1985), 429-435.

[R5] Multidimensional inverse scattering problems, Longman, New York, 1992 (expanded Russian edition will be published by Mir, Moscow, 1993). 
[RZ1] A. G. Ramm and A. Zaslavsky, Reconstructing singularities of a function from its Radon transform, Technion, preprint 1992.

[RZ2] - Asymptotic behavior of the Fourier transform of a piecewise smooth function, Technion, preprint 1992.

[RSZ] A. G. Ramm, A. Steinberg and A. Zaslavsky, Stable calculation of the Legendre transform, Technion, J. Math. Anal. Appl. (to appear).

[T] R. Thom, Sur la théorie des enveloppes, J. de Math. Pure Appl. 56 (1962), 177-192.

[Z] A. I. Zaslavsky, Dual varieties and Legendre transforms, submitted.

[Zl] V. A. Zalgaller, The theory of envelopes, Nauka, Moscow, 1975. (Russian)

Mathematics Department, Kansas State University, Manhattan, Kansas 665062602

E-mail address: ramm@ksuvm.ksu.edu

Department of Mathematics, Technion-Israel Institute of Technology, 32000 HAIFA, ISRAEL

E-mail address: mar9315@technion.technion.ac.il 